\title{
Lesson of the month 2: Non-uraemic calciphylaxis - an unexpected differential diagnosis for a necrotic ulcer
}

\author{
Authors: Edward J Bataillard, ${ }^{\mathrm{A}}$ Joanna Clayton, ${ }^{\mathrm{B}}$ Jonathan Riordan ${ }^{\mathrm{C}}$ and Thomas Galliford ${ }^{\mathrm{D}}$
}

\section{Calciphylaxis is an uncommon cause of skin necrosis seen almost exclusively in patients with end-stage renal disease. We present an unexpected diagnosis of calciphylaxis in a patient with normal renal and parathyroid function. The patient presented with a month-long history of painful bilateral necrotic leg ulcers, resistant to conventional treatment. She developed severe sepsis requiring admission to the intensive care unit, and despite escalation of antibiotic therapy and meticulous wound care management, eventually died. A biopsy confirmed a diagnosis of calciphylaxis. We suspect that warfarin therapy may have contributed to the development of this condition. Through this case we aim to raise awareness of calciphylaxis as a differential diagnosis of non-healing necrotic skin ulcers, especially in patients with known risk factors including established warfarin therapy.}

KEYWORDS: Non-uraemic, calciphylaxis, necrotic ulcer, skin necrosis, warfarin

\section{Case history}

A 56-year-old Caucasian female presented from a rehabilitation centre to a general medical ward. She had a month-long history of a painful left posteromedial thigh ulcer, which had significantly limited her mobility to the extent that she had become bedbound. She had received several courses of antibiotics as treatment for cellulitis associated with what was initially considered to be a pressure ulcer, however her condition had deteriorated.

The patient was morbidly obese (BMI, $44 \mathrm{~kg} / \mathrm{m}^{2}$ ). Her past medical history included atopic eczema, recurrent cellulitis, lymphoedema, adrenal insufficiency secondary to topical steroid use, and recurrent unprovoked pulmonary embolism. Her drug history included warfarin, started six months previously (target

Authors: ${ }^{A}$ core medical trainee, West Hertfordshire Hospitals NHS Trust, Watford, UK; ${ }^{B}$ core medical trainee, West Hertfordshire Hospitals NHS Trust, Watford, UK; ${ }^{C}$ core medical trainee, West Hertfordshire Hospitals NHS Trust, Watford, UK; ${ }^{\mathrm{D}}$ consultant endocrinologist and physician, West Hertfordshire Hospitals NHS Trust, Watford, UK

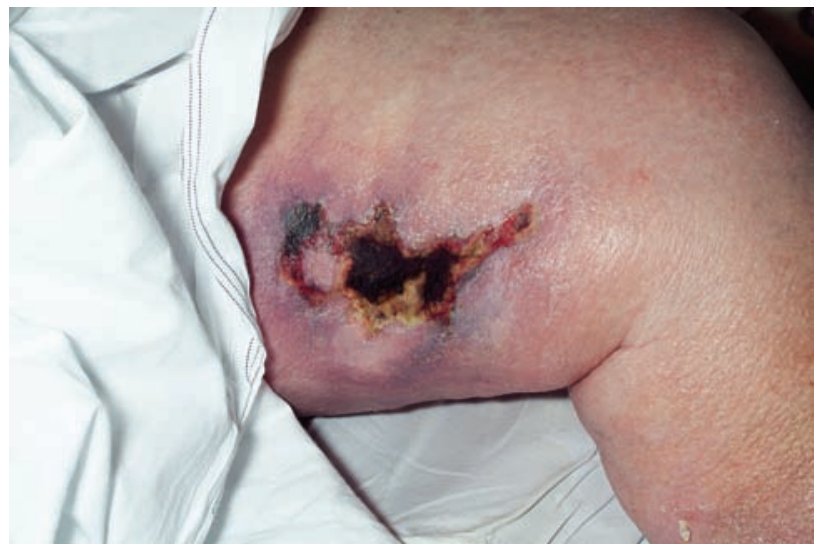

Fig 1. Extensive ulceration on the left thigh with necrosis and eschar formation.

international normalised ratio (INR), 2.5), oral hydrocortisone and prophylactic penicillin $\mathrm{V}$ for recurrent cellulitis.

On admission she was afebrile with stable haemodynamic observations. She had a large tender stellate violaceous lesion with a necrotic centre and surrounding warmth and erythema. The lesion was approximately $3 \times 4 \mathrm{~cm}$ in size, situated on the posteromedial aspect of her left thigh (Fig 1).

\section{Investigations}

Admission blood tests revealed raised inflammatory markers (white cell count $11 \times 10^{9} / \mathrm{L}$ (reference range 3.6-11.0 $\times 10^{9}$ ), C-reactive protein $228 \mathrm{mg} / \mathrm{L}$ (reference $<5.0 \mathrm{mg} / \mathrm{L}$ )), low albumin at $20 \mathrm{~g} / \mathrm{L}$ (reference range 35-50), normal renal function (urea $2.8 \mathrm{mmol} / \mathrm{L}$, creatinine $41 \mu \mathrm{mol} / \mathrm{L}$ ), normal bone profile and parathyroid function (corrected calcium $2.43 \mathrm{mmol} / \mathrm{L}$, phosphate $1.43 \mathrm{mmol} / \mathrm{L}$, parathyroid hormone $3.6 \mathrm{pmol} / \mathrm{L}$ ), and an INR of 2.8. A creatine kinase level and autoimmune screen were also normal.

Magnetic resonance imaging excluded osteomyelitis. Wound swabs revealed heavy growth of Pseudomonas species and antibiotics were escalated to piperacillin and tazobactam. A biopsy was taken which revealed epidermal necrosis with underlying congestion and inflammation. The small and medium sized vessels showed extensive calcification, consistent with a diagnosis of calciphylaxis (Fig 2). 


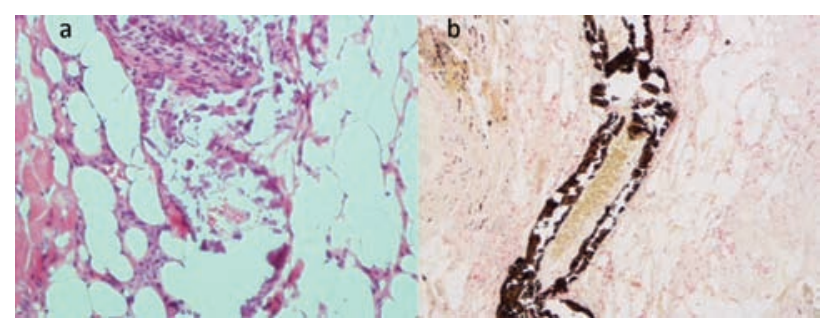

Fig 2. Skin biopsy histology. (a) Calcification in blood vessels ( $\times 20$ magnification, H\&E stain); (b) von Kossa stain highlighting calcium in blood vessel (×10 magnification).

\section{Treatment}

Despite treatment the ulcer grew and became more necrotic, and a second ulcer developed on her right thigh. Antibiotic therapy was escalated and a wound care programme was commenced including betamethasone cream and compression bandages.

On day 15 of her admission the patient became febrile, tachycardic with atrial fibrillation, and hypotensive. She was admitted to the intensive care unit for further management of septic shock. Her treatment included (i) broad spectrum antibiotics, including daptomycin and gentamicin; (ii) reversal of warfarin with prothrombin complex concentrate and discontinuation of warfarin therapy; (iii) blood transfusion to correct normocytic anaemia; (iv) human albumin solution to correct hypoalbuminaemia; and (v) intravenous infusions of sodium thiosulphate.

Although the patient initially stabilised after 10 days on the intensive care unit, the ulcers became larger, more necrotic and erythematous (Fig 3). They spread to her buttocks and abdomen. She was deemed not fit for surgical debridement. A decision was made for no further escalation and she was palliated until her death 7 weeks after admission.

\section{Discussion}

Calciphylaxis is a highly morbid and often lethal condition which results in cutaneous necrosis. It is characterised

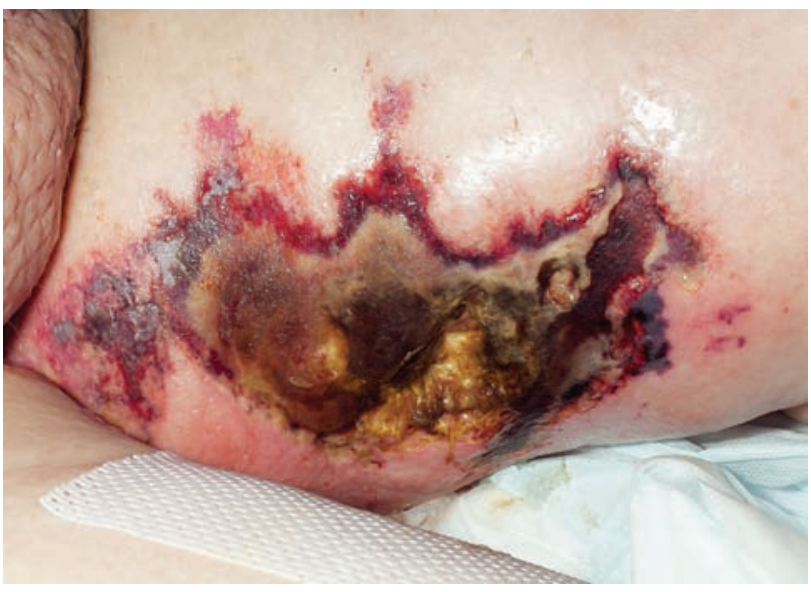

Fig 3. Worsening necrosis and erythema of posteromedial thigh lesion.

Table 1. Causes of and factors predisposing to NUC.

\section{Causes of NUC}

Hyperparathyroidism

Malignancy

Alcoholic liver disease

Connective tissue disease

Diabetes mellitus

Crohn's disease

Vitamin D deficiency

Chemotherapy-induced protein C and $\mathrm{S}$ deficiency

Rapid weight loss

Chronic kidney disease (not end stage)

NUC $=$ non-uraemic calciphylaxis

by extensive dermal vascular calcification and occlusion with resultant infarction of the skin and subcutis. The pathophysiology of the condition is incompletely understood, however it is a recognised complication of end-stage chronic kidney disease, with a prevalence of up to $4 \%$ in haemodialysis patients. ${ }^{1}$ Although the disease has been reported in patients with normal renal function (so-called non-uraemic calciphylaxis (NUC)), its incidence is rare. A 2008 systematic review identified just 35 cases, ${ }^{2}$ and at least 30 more have since been described.

As a result, although calciphylaxis is more easily suspected in vulnerable patients with end-stage renal failure by nephrologists who are better acquainted with the condition, it poses a significant diagnostic challenge when it occurs in nonuraemic patients. Moreover, the causes of NUC are varied and inconsistent. To date, the two major aetiologies identified are hyperparathyroidism (28\%) and malignancy (22\%), as well as a number of other unrelated causes (Table 1). ${ }^{2}$

Our patient possessed a number of predisposing risk factors. These included obesity, warfarin therapy, hypoalbuminaemia, systemic corticosteroid use, and being in the at-risk patient demographic (Caucasian middle-aged female).

The association with warfarin therapy is well established. The underlying mechanism is believed to be related to inhibition of vitamin K-dependent carboxylation of an extracellular protein known as matrix G1A protein (MGP). ${ }^{3}$ This results in a state of pro-calcification, which is supported by studies in mouse models wherein disruption of MGP leads to extensive vascular calcification. $^{4}$

However, warfarin may add diagnostic uncertainty due to confusion with the unrelated phenomenon of warfarin-induced skin necrosis (WISN). Indeed, we initially suspected a possible diagnosis of WISN as a cause of the patient's necrotic ulcers. We note that there have been several case reports of similar initial diagnostic confusion. ${ }^{5,6}$ Both conditions may have similar macroscopic appearances, and similar regions of the skin may be affected, with areas of high adiposity being most at risk. The key difference in presentation is related to the timeline of warfarin therapy. The majority of WISN occurs at the onset of 
warfarinisation, as early as day 1 , peaking at day 3 , and rarely occurring beyond day 10. In contrast, warfarin therapy is often long established when it is associated with calciphylaxis.

Treatment options for calciphylaxis include management of concurrent infection, treatment of hyperparathyroidism and removal of sensitising factors. There have also been reported cases of successful recovery with intravenous sodium thiosulphate, which is a potent reducing agent and is emerging as an important experimental treatment option. Successful treatment with hyperbaric oxygen has also been reported. Due to the uncertainty of management options however a multi-intervention approach is often adopted. ${ }^{7,8}$

Given the high mortality associated with NUC (reported to be $>50 \%$ ), it is possible that many cases are undiagnosed before death, or misdiagnosed, attributed instead to non-healing leg ulcers of other aetiology. This highlights the importance of raising awareness of the condition, especially given that novel treatment strategies exist for the disease. We encourage general physicians to consider NUC as a differential diagnosis in patients with an established cause or known risk factors.

\section{References}

1 Angelis M, Wong LL, Mysers SA, Wong LM. Calciphylaxis in patients on haemodialysis: A prevalence study. Surgery 1997;122:1083-9.
2 Nigwekar SU, Wold M, Sterns RH, Hix JK. Calciphylaxis from nonuremic causes: a systematic review. Clin J Am Soc Nephrol 2008;3:1139-43.

3 Lomashvili KA, Wang X, Wallin R, O'Neill WC. Matrix GLA protein metabolism in vascular smooth muscle and role in uremic vascular calcification. J Biol Chem 2011;286:28715-22.

4 Luo G, Ducy P, McKee MD et al. Spontaneous calcification of arteries and cartilage in mice lacking matrix GLA protein. Nature 1997;386:78-81.

5 Chacon G, Nguyen T, Khan A et al. Warfarin-induced skin necrosis mimicking calciphylaxis: a case report and review of the literature. J Drugs Dermatol 2010;9:859-63.

6 Breakey W, Hall C, Vann Jones S et al. Warfarin-induced skin necrosis progressing to calciphylaxis. J Plast Reconstr Aesthet Surg 2014;67:244-6.

7 Fernandes C, Maynard B, Hanna D. Successful treatment of calciphylaxis with intravenous sodium thiosulfate in a nonuremic patient: case report and review of therapy side effects. J Cutan Med Surg 2014;18:356-60

8 Baldwin C, Farah M, Leung M et al. Multi-intervention management of calciphylaxis: a report of 7 cases. Am J Kidney Disease 2011;58:988-91.

Address for correspondence: Dr T Galliford, Watford General Hospital, Vicarage Road, Watford WD18 OHB, UK.

Email: thomas.galliford@whht.nhs.uk

\title{
Image of the month: The Mount Fuji sign
}

\author{
Authors: Karan Manoj Anandpara, ${ }^{\mathrm{A}}$ Yashant Aswani ${ }^{\mathrm{B}}$ and Priya Hira ${ }^{\mathrm{C}}$
}

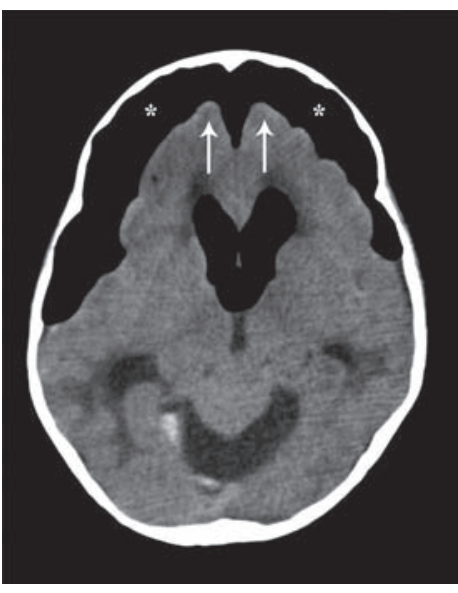

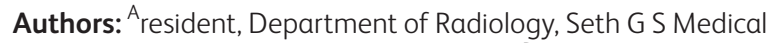
College and KEM Hospital, Mumbai, India; ${ }^{\text {B }}$ resident, Department of Radiology, Seth G S Medical College and KEM Hospital, Mumbai, India; ' associate professor, Department of Radiology, Seth G S Medical College and KEM Hospital, Mumbai, India
Fig 1. Axial unenhanced computed tomography images of the brain demonstrates bilateral subdural hypoattenuating areas $\left(^{*}\right)$ causing compression of the frontal lobes. Note the widening of the interhemispheric space between the tips of the frontal lobes (white arrows) forming the characteristic silhouette of Mount Fuji.

A 6 year-old-girl developed decreased responsiveness 12 hours after surgical resection of a pilocytic astrocytoma by posterior fossa craniotomy. Computed tomography of the brain demonstrated the 'Mount Fuji sign' (Fig 1). This sign refers to hypoattenuating subdural air that causes compression and separation of bilateral frontal lobes suggestive of underlying tension pneumocephalus and warrants emergent neurosurgical reference. ${ }^{1}$.

\section{References}

1 Michel SJ. The Mount Fuji sign. Radiology 2004;232:449-50.

Address for correspondence: Dr KM Anandpara, Department of Radiology, Seth G S Medical College and KEM Hospital, Acharya Donde Marg, Parel, Mumbai 400012, India. Email: karananandpara@gmail.com 